\title{
Composition, properties and potential food applications of natural emulsions and cream materials based on oil bodies
}

\author{
Constantinos V. Nikiforidis, Anthia Matsakidou and Vasilios Kiosseoglou*
}

Received 31st January 2014

Accepted 14th May 2014

DOI: $10.1039 / \mathrm{c} 4 \mathrm{ra00903g}$

www.rsc.org/advances

\begin{abstract}
Oil bodies are micron- or submicron-sized organelles found mainly in parts of plants such as seeds, nuts or some fruits and their main role is to function as energy stores. Their structure is made up of a core of triglycerides covered by a protein-phospholipid layer which protects the oil bodies against external chemical/mechanical stresses. Following treatment with aqueous media of the rich-in-oil raw materials, an extract of oil bodies, dispersed in a solution of exogenous plant proteins, is obtained. Effective recovery of oil droplets from the initial extract, which is in effect a relatively dilute natural emulsion, leads to the preparation of either a more concentrated natural emulsion with a composition in terms of oil and protein close to that of animal milk or, alternatively, to a concentrated oil droplet-based "cream". Both the natural emulsion and the "cream" can be exploited in the development of a number of novel food products by suitably substituting the oil/fat droplets of the traditionally-prepared food product with natural oil droplets.
\end{abstract}

\section{Introduction}

Oleaginous plants store energy in the form of triacylglycerols mainly in their seeds or nuts. Triacylglycerols in the cells of the plant energy reserves are found in the core of organelles called oil bodies, oleosomes or spherosomes, ${ }^{1}$ along with the bigger in size protein bodies. ${ }^{2,3}$ Oil bodies exhibit unique physical and chemical stability due to the presence at their surface of a mixed layer of phospholipids and hydrophobic proteins such as oleosin, despite the stressful environmental conditions to which they are usually exposed, protecting thus the triacylglycerols until the time of germination comes. ${ }^{1}$

The oil bodies found in the various plant materials present more or less similar structural characteristics, irrespective of their origin. Soybeans, ${ }^{1}$ maize germs,${ }^{1,4}$ wheat germs, ${ }^{1}$ oats, ${ }^{5}$ sunflower seeds, ${ }^{6}$ pumpkin seeds, ${ }^{7}$ sesame seeds, ${ }^{8}$ rice, ${ }^{1}$ rapeseeds ${ }^{1}$ and nuts like almonds, ${ }^{9}$ peanuts, ${ }^{8}$ adlay ${ }^{10}$ and Brassica napus $^{11}$ are some of the materials where oil bodies have been identified and studied due to their technological importance for the food or other industries.

Oil bodies can be extracted from plant materials by using aqueous media in the place of the conventional oil-extracting organic solvents, mainly hexane. ${ }^{12}$ The key difference between the novel aqueous extraction of oil bodies and the conventional one is that in the case of the first approach an oil-in-water emulsion, based on intact or partially disrupted oil bodies, and

Laboratory of Food Chemistry and Technology, Department of Chemistry, Aristotle University, Thessaloniki, Greece, GR-54124. E-mail: kiosse@chem.auth.gr; Fax: +30231-0-997779; Tel: +30-231-0-997834 not a solution of oil in an organic solvent is obtained. As a result, aqueous extraction presents a number of significant benefits compared to the conventional extraction method. First, there are some important benefits from expunging organic solvents from the vegetable oil extraction process, connected with technical issues relevant to environmental safety and health concerns. US National Institute for Occupational Safety and Health (NIOSH) has classified hexane as a flammable and, under given circumstances, as an explosive solvent. ${ }^{13}$ There have been numerous incidents of fire or explosion in soybean factories $^{14,15}$ and special precautions, concerning hexane Also, long time occupational exposure to hexane may affect the human nervous system ${ }^{13}$ and the European Union has established maximum hexane residue limits for various food products. ${ }^{16}$ Secondly, since very often the vegetable oils have to be incorporated into emulsion food products, energy consuming and highly expensive homogenization process has to be performed prior to their incorporation. Extraction of oil in the form of oil bodies' emulsion has the advantage of obtaining a product that is already naturally emulsified. Moreover, this natural emulsion may exhibit remarkable stability and high nutritional value because it does not have to be subjected to any refining applied to the raw material a simultaneous protein co-extraction also takes place. The extracted protein can be then exploited in the preparation of protein isolates or concentrates and the same holds for the solid residue of the extraction. These benefits of aqueous extraction of oil bodies from plant materials management to ensure employees labor safety, must be taken. treatment. Finally, when aqueous extraction of oil bodies is 
reveal the emerging opportunities for the industry to expand into new markets and benefit financially.

This review first concentrates on the description of the role of oil bodies in the plant cells and provides a general description of their structure and composition. What follows is the investigation of the possible mechanisms behind aqueous oil body extraction and the processes available to achieve optimized extraction and oil body recovery. Next, emphasis is given on the physical and chemical properties of oil droplets in their aqueous extracts that may affect the preparation of possible food or other products. The review is concluded with a discussion on the potential of exploiting the recovered oil bodiesbased preparations as ingredients in the development of novel food products.

\section{Role, morphological characteristics and composition of oil bodies}

\subsection{Role in plants and morphological characteristics}

The oil bodies were first described by Hubener ${ }^{17}$ in his description for a leafy liverwort as transparent droplets with a shining, membranous texture. The nature of these bodies could not be recognized, but the author suggested that they were diluted starch. They were later named cell bodies (Zellen Körper) ${ }^{18}$ and afterwards ${ }^{19}$ cell vesicles. In $1874,{ }^{20}$ the spherical structures that could be viewed in liverworts were named oil bodies (Ölkörper) due mainly to their fatty nature. Some earlier workers recognized a genuine membrane surrounding the oil bodies. Their structure was confirmed by electron microscope studies but there were a lot of differing opinions concerning the nature of the membrane. ${ }^{21}$ A number of researchers ${ }^{19,20,22}$ recommended that the membrane surrounding the bodies was a genuine one while some others ${ }^{23-25}$ assumed that it was an artifact produced by fixatives. On the other hand, Garjeanne ${ }^{\mathbf{2 6}}$ claimed that the visible ring around the oil is tanninized protein. Later studies and observations of oil bodies with electron microscopy, indicated that the membrane was a monomolecular layer of phospholipids, arranged in such a way as to prevent adjacent bodies from coalescing. ${ }^{27,28}$ It was only a couple of years later that researchers found out that isolated oil bodies were comprised of triglycerides, phospholipids and proteins which were completely bonded on the particles. ${ }^{29}$ It was also reported that the ratio between triglycerides, phospholipids and proteins in the oil bodies varied quite widely among different oleaginous seeds.

Later research on plant oil bodies provided evidence that the proteins were integral constituents of the particles, probably localized entirely at the surface. ${ }^{30}$ These proteins may be connected with the oil bodies' physical and chemical stability, or become involved in interactions with other organelles. ${ }^{31,32}$ Intracellular oil bodies, were assumed to serve as energy storage reserves to support periods of active metabolism such as seedling growth during germination. ${ }^{33}$ Since, however, they cover up to $75 \%$ of the seed's volume and are present in almost all plant cells and not just in storage tissues, they may also have other intracellular functionalities. ${ }^{34}$ Earlier research proved that oil bodies are dynamic organelles ${ }^{35}$ that are actively involved in cellular lipid homeostasis and energy metabolism, ${ }^{36}$ so they are important in many physiological or pathological situations. ${ }^{37}$

Oil bodies vary in size from nanoscale to a few $\mu \mathrm{m} .^{38,39}$ Environmental factors, along with the surface protein content, play an important role in determining their initial shape and size. According to cryo-SEM analysis, the oil bodies in seeds with a high moisture content, like sunflower seeds (>14.0 wt\%), appear to have a spherical shape, in contrast to seeds with low moisture, like maize germ $(<7.0 \mathrm{wt} \%)$, where the oil bodies have irregular shapes, depending on the available space (Fig. 1). ${ }^{\mathbf{4 0}}$ These findings indicate that the protein membrane that covers and stabilizes the particles is very elastic. As mentioned above, apart from environmental factors, the size of the particles is mainly dictated by the ratio between the oil and the interfacial protein content. ${ }^{41}$ In general, oleaginous seeds with relatively small-sized oil particles appear to exhibit a rather low ratio of triglycerides to interfacial proteins as compared to seeds with oil bodies of large size. ${ }^{42}$ Oil bodies from olives and avocados, which have very low surface protein contents, poorly exhibit physical stability. ${ }^{\mathbf{4 3 , 4 4}}$

Apart from their important biological function the interfacial proteins, together with the phospholipids, contribute to the physicochemical stability of the oil droplet surface. ${ }^{45,46}$ Treatment of oil bodies with trypsin may lead to the complete rupture of the surface membrane, while treatment with phospholipase A2 or C does not appear to induce any changes. ${ }^{46}$ These findings indicate that the phospholipids at the oil bodies' surface are probably entangled with the surface protein molecules with the latter forming a layer upon the phospholipids that does not allow the enzymes to have access to their hydrophilic head.
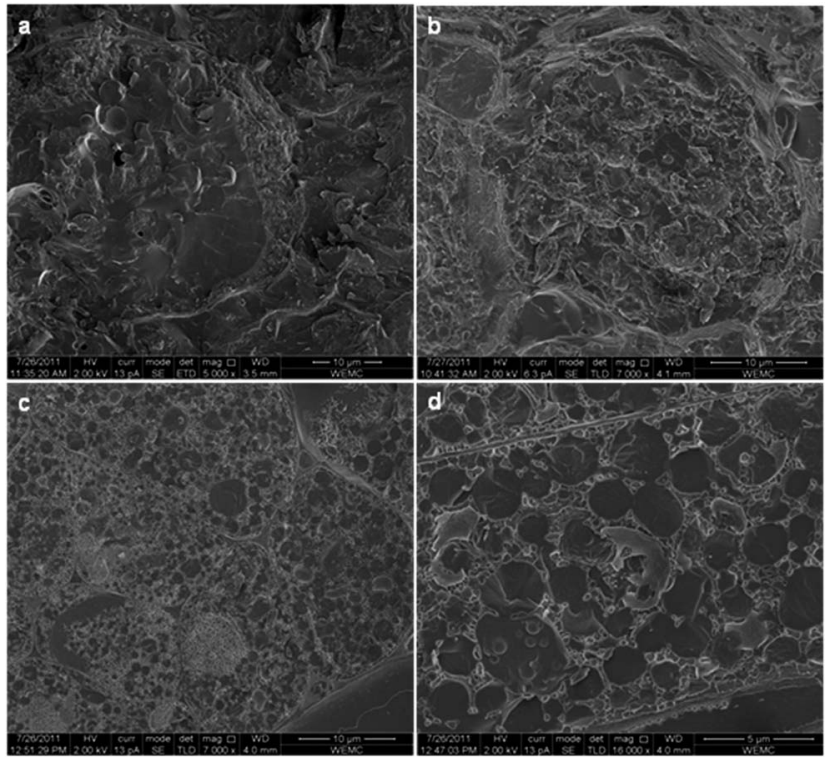

Fig. 1 Cryo-SEM images of maize germ hydrated for 1 day (a) and 2 days (b) and of sunflower seeds hydrated for $1 \mathrm{~h}$ and magnified 7000 (c) or 16000 (d) times. Reprinted from Nikiforidis et al. ${ }^{40}$ 
Oil bodies in the cells are remarkably stable against aggregation and coalescence. In this way, the seeds can withstand environmental stresses for long periods without the appearance of large physical or chemical changes in their oil bodies. ${ }^{47}$ This stability could be attributed to the relatively high negative charge of the oil bodies' surface or to steric repulsions that prevent the oil bodies from coming too close and aggregate or coalesce. On the other hand, an additional factor that is also likely to prevent oil-body coalescence is the high viscosity of the environment in the dry seed (cytoplasm). ${ }^{48}$

\subsection{Composition}

As has been previously mentioned, the oil bodies have a triglyceride core that is covered with a mixed membrane of proteins and phospholipids. Depending on the source, the triglyceride's content of the oil bodies is around 94.0-98.0 wt\% while the phospholipids and proteins content is $0.6-2.0 \mathrm{wt} \%$ and $0.6-3.0 \mathrm{wt} \%$, respectively. Oil bodies' proteins have long been characterized either as structural proteins or enzymes. However, recent research studies identified other groups of proteins associated with oil bodies. ${ }^{49}$ The oil body surface proteins are distinguished from other proteins by their extended central hydrophobic domain. They are categorized into oleosins, caleosins and steroleosins, with oleosins being the dominant surface proteins. ${ }^{39,50}$

Oleosins are hydrophobic proteins with a molecular mass of about 15 to $26 \mathrm{kDa}$ and a molecule that has an uninterrupted central hydrophobic domain of about 70 amino acid residues. ${ }^{51}$ Oleosins probably adopt a unique conformation at the oil body surface, creating a membrane where a large hydrophobic domain is flanked by two hydrophilic domains. The hydrophobic domain is assumed to be buried within the triacylglycerol core. The C-terminal domain is located on the oil body surface while the $\mathrm{N}$-terminal domain may contain a mixture of structures and is located in the cytoplasm that faces the cytosol. ${ }^{52}$

Caleosin (25-35 kDa) and steroleosin (40-55 kDa) molecules have a similar but shorter hydrophobic sequence and longer hydrophilic domains that are located on the oil body surface or oriented towards the cytosol. ${ }^{50,53}$

Oil bodies have very interesting and useful characteristics for a wide range of applications in colloid science. Their extraction, therefore, as well as the physical and chemical properties of their extract is a topic of increasing interest.

\section{Aqueous extraction of oil bodies}

Investigation of the mechanisms of aqueous oil body extraction from plant raw materials and the development of optimized methods and procedures for their extraction/recovery is a relatively recent area of research, prompted by technological and economic needs mainly of the food industry. Since, however, this extraction approach is a novel one, for the industry to invest in this new technology extensive and, probably, costly adjustment of its infrastructure may be needed. Hence, technological implementation of the procedure, high efficiency, quality and the possibility of finally extracted product's exploitation are extremely crucial parameters for the viability of such a project and have to be thoroughly investigated.

Several studies have focused on explaining the mechanisms behind aqueous extraction of oil bodies and also on developing extraction processes and optimizing the parameters of aqueous extraction and recovery of oil bodies from their initial aqueous extract. Tzen and Huang ${ }^{1}$ put forward a method of isolating oil bodies from plant seeds in the form of a natural emulsion, to study the oil droplet surface layer composition and structure. However, the first attempt to extract oil bodies with the use of aqueous methods was described by Rhee et al. ${ }^{54}$ who studied the effect of extraction process parameters on oil yield.

\subsection{Aqueous extraction mechanisms}

As was previously described, oil bodies, which contain the oil in seeds, beans or nuts, fill the space between protein bodies, which occupy most of the cell space and they are both embedded into the cytoplasmic network. Plant cell walls consist of cellulose, hemicelluloses, lignin and pectin (Fig. 2). ${ }^{55}$ The wall allows mass transfer through plasmodesmata, which are openings of 20 to $80 \mathrm{~nm}$ in diameter, allowing molecules of a mass of up to $9 \mathrm{kDa}$ to pass through. ${ }^{56}$ As a result, extraction of vegetable oils is based on the diffusion of oil constituents to the solvent, when the rich-in-oil material, usually in the ground or flaked form to maximize the exposure of oil to the solvent, is brought into contact with the extraction medium..$^{57}$ In addition to mechanical means, cell wall disruption is also possible by using enzyme mixtures, consisting of cellulases, hemicellulases, pectinases, and even proteases. ${ }^{55}$

During conventional organic solvent extraction, oil dissolves into the solvent, following the disruption of the cell walls, while the proteins remain in the meal along with the carbohydrates and the fiber. Aqueous extraction, on the other hand, is a quite different mechanism as simultaneous extraction of both the oil and the water-soluble proteins takes place. ${ }^{58}$ Oil bodies are released into the aqueous medium, as soon as the proteins diffuse first, provided that there is an adequate amount of solvent. Since the protein body aggregates are easily disrupted

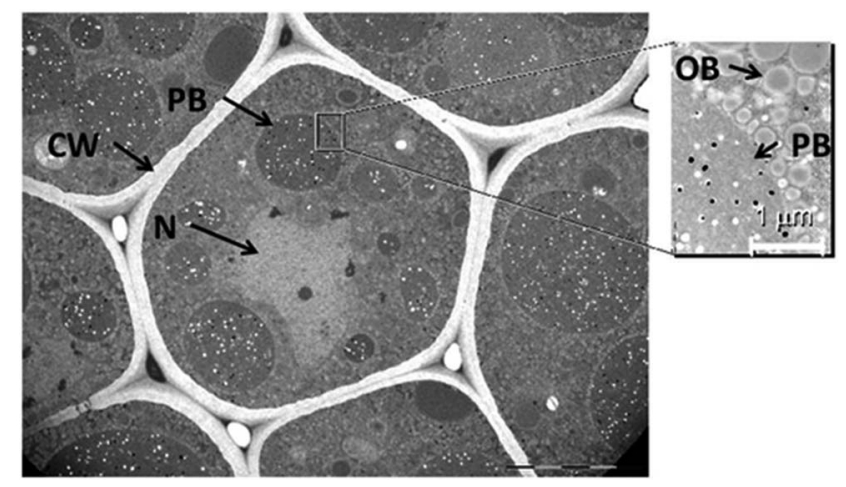

Fig. 2 TEM of soybean cotyledon cell cross-section. PB, protein body; CW, cell wall; N, cell nucleus; OB, oil body. Reprinted with permission from Campbell et al. ${ }^{2}$ 
and removed by the water, extensive damage of the cellular consistency may take place leaving behind a wide path for the oil bodies to pass through. ${ }^{59}$

Agitation, according to Campbell and Glatz, ${ }^{2}$ apart from its role in the disruption of the cellular barriers, may also lead to the disruption of oil bodies aggregates easing in this way their escape during extraction. These authors, however, reported that during extraction of soybean oil, coalescence between the smallsized oil bodies into larger ones may take place, to such a size that makes it difficult for them to diffuse. Hence, agitation has to provide enough energy to effect the reduction of the size of larger droplets and increase their mobility. In addition, in the case of application of extrusion, heat and pressure are exercised upon the soybeans resulting in a decrease of the protein solubility to such an extend as to block the release of the oil from the extracellular protein matrix, even though there is much more extended cellular disruption compared to that of soy flour. In case of applying extrusion to soybeans, protease could play an important role in increasing the yield of oil extraction, since proteolysis dissolves insoluble denatured proteins. ${ }^{2}$

The oil extracted with an aqueous solvent is recovered either as a creamed, separated phase or in the form of an oil-in-water emulsion, both made up of intact or partially disrupted oil bodies, unlike the product resulting from conventional extraction. ${ }^{55}$ Given the above, although oil extractability with the aid of aqueous media depends on parameters, such as the degree of the raw material comminution, solid-to-solvent ratio, extraction time and temperature, which are also critical parameters for conventional oil extraction, it may also depend on parameters that relate directly to protein extractability. These parameters are protein solubility, $\mathrm{pH}$ value and salt concentration.

\subsection{Process steps}

Most workers involved in research of aqueous oil extraction follow more or less the same process steps to recover the oil either in the form of oil droplets or pure oil. This review mainly focuses on the description of the process of aqueous oil extraction in the form of intact or partially disrupted oil bodies, displayed in Fig. 3., ${ }^{\mathbf{1 , 3 6 0}}$ Conventional solvent extraction of richin-oil plant materials usually involves the application of successive washing steps with hexane after subjecting the raw material to some mechanical treatment to reduce the particle size. The solvent is finally recovered from the extracted oil and reused. On the other hand, the aqueous oil body extraction process includes the steps of raw material mechanical pretreatment, extraction, filtration and centrifugation.

Pretreatment of plant material (Fig. 3), before the main stage of aqueous extraction, usually involves grinding followed by water soaking of the comminuted raw plant material. ${ }^{\mathbf{1 , 3 5 4 , 5 8}}$ Grinding provides better exposure of oil bodies to the water as a result of cell structure rupture and hence improved extractability. Moreover, hydration allows the water molecules, especially in materials of low moisture content, like maize germ, to penetrate into the cell network, allowing more efficient extraction. ${ }^{58}$ Hydration may last several hours. The importance of solid to water ratio applied was stressed by Campbell and Glatz,

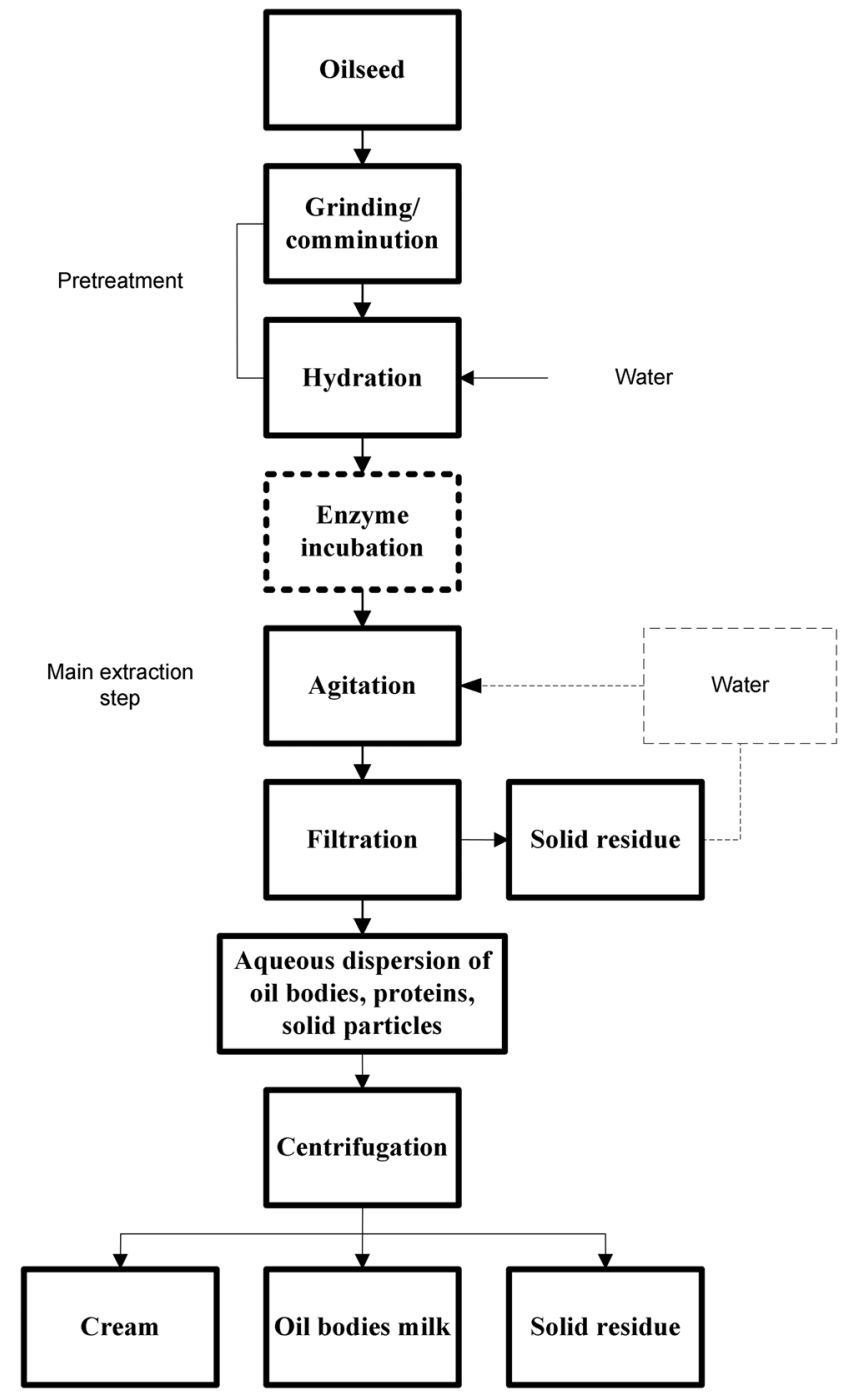

Fig. 3 Process diagram of aqueous extraction of oil bodies.

de Moura and Johnson ${ }^{61}$ and Rhee et al. ${ }^{54}$ Their work showed that increasing solid to water ratio may lead to substantial improvement of the extraction yield.

The main extraction stage which follows mechanical pretreatment of the raw material involves homogenization, ${ }^{\mathbf{1 , 1 2}}$ agitation, ${ }^{3}$ grinding ${ }^{62,63}$ or treating the wet slurry in a colloid mill. ${ }^{63}$ Rosenthal et al. ${ }^{58}$ reported that an increase in the agitation rate caused an initial increase in the yield of soybean oil extraction, before most of the oil-bearing cells were ruptured. After that point, further increase in agitation rate had a limited effect on the yield value. Similarly, long time duration of the extraction step led to higher extraction yield values. ${ }^{2}$

As has been previously mentioned, during the extraction stage protein diffusion into the water takes place, with the simultaneous liberation of the oil bodies. As a result, the solubility of the plant material proteins plays a key role for effective oil body extraction. Therefore, parameters such as the presence or absence of salt, $\mathrm{pH}$ value and temperature, are crucial in determining the yield of extraction. 
Sodium chloride addition may be needed in order to effect the solubilization of the proteins and thus aid in the release of the oil bodies from the cell network. ${ }^{1}$ In addition, the structural damage of the cells, due to the increased osmotic pressure of the sodium chloride solution, may lead to increased solid particle surface in contact with the solvent, resulting in higher extraction efficiency. ${ }^{64}$ The extraction yield is also maximized when the extraction is performed under alkaline conditions due to the increased solubility of the vegetable proteins. ${ }^{3,58,63}$ When aqueous extraction of peanut oil bodies was performed at $\mathrm{pH}$ 4.0, partial destabilization of their structure took place leading to the appearance of a clear oil phase at the top of the extract. ${ }^{54}$ On the other hand, Nikiforidis and Kiosseoglou ${ }^{3}$ reported that the higher than expected dispersibility and extractability of maize germ oil bodies at pH 6.0, which is a value very close to that of the isoelectric point of the maize germ oleosomes, should be attributed to the presence of storage proteins at the surface of oil bodies along with oleosins. Storage proteins have more acidic character compared to the latter. The presence of these proteins may have altered the surface charge of the oil bodies, thus changing the oil body dispersibility-dependence on $\mathrm{pH}$. Regarding the influence of extraction temperature, the reduced yield of soybean oil extraction, when the temperature was over $50{ }^{\circ} \mathrm{C}$, was attributed to denaturation of the proteins, leading to the decrease of their solubility and hence extractability. ${ }^{58}$ In straight contrast to the above, Rhee et al. ${ }^{54}$ concluded that the temperature of $60-64{ }^{\circ} \mathrm{C}$ is required to maximize extraction of peanut oil.

The crude product of the extraction is a mixture of solid residue particles and oil droplets dispersed in a protein solution (Fig. 3) that has to be filtered through multiple layers of cheesecloth in order to remove the dispersed solid particles. ${ }^{\mathbf{1}}$ The solid residue of the extraction, consisting mainly of cellulose, insoluble proteins and non-extracted oil bodies, may have to be subjected to further extraction treatment in order to increase the yield of

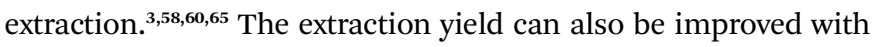
the aid of enzymes. During the process of aqueous enzymatic extraction, a step of treatment for enzyme activation, involving pH adjustment and the thermal incubation (Fig. 3) according to optimum standards of each enzyme used, is applied to facilitate cell lyses. ${ }^{60,66}$ The enzyme level, time and liquid to solid ratio were found to affect to a significant extent the extraction yield of oil from bayberry kernels. ${ }^{67}$ Similar findings were reported by Xie et $a .^{68}$ for the aqueous enzymatic extraction of wheat germ oil. Finally, Kapchie et $a .^{64}$ suggested that a mixture of cellulose, pectinase and hemi-cellulolytic enzymes, was the most effective in hydrolyzing the cell walls of soybean.

\subsection{Yield of extraction process}

The most frequently mentioned drawback of applying aqueous oil extraction to plant materials is the low yield of the process when compared with the yield of the processes based on the use of an organic solvent. A review of the available literature, however, revealed that a sufficiently high yield can be reached when the aqueous extraction parameters are suitably manipulated.
Rhee et al. ${ }^{54}$ was the first to our knowledge who succeeded in achieving an almost complete aqueous extraction of peanut oil in the form of oil bodies ( $98.7 \pm 0.7 \mathrm{wt} \%)$ by careful manipulation of critical extraction parameters such as the degree of grinding, solid to solvent ratio value, extraction time and extraction temperature. As was concluded, extraction of finely ground peanuts for $30 \mathrm{~min}$ at a solid to solvent ratio of $1: 6$ and a temperature of $60-64{ }^{\circ} \mathrm{C}$, resulted in an oil yield of approximately $96 \%$. An extraction yield of $95.3 \mathrm{wt} \%$ for maize germ oil was achieved by Nikiforidis and Kiosseoglou ${ }^{3}$ following the application of three repeated extraction cycles to a finely comminuted maize germ material at $\mathrm{pH}$ 9.0. de Moura et al. ${ }^{69}$ reached a very high yield (93-97 wt\%) following extraction of oil from full-fat soybean flakes by implementing an enzyme-assisted aqueous extraction process using an endoprotease. In another parameter-optimization study, Li et al. ${ }^{70}$ reported that the yield of $87 \%$ of wheat germ oil extraction can be achieved by applying an enzymatic aqueous extraction method at a water to wheat germ ratio of $3.5\left(\mathrm{v} / \mathrm{w}, \mathrm{ml} \mathrm{g}^{-1}\right)$, a $\mathrm{pH}$ value 5.0 , a temperature of $48.5^{\circ} \mathrm{C}$ and an extraction time of $6 \mathrm{~h}$. The use of a mixture of pectinase, cellulase and $\beta$-gluconase brought about an increase of the yield of soybean oleosomes extraction to a value as high as $63.23 \mathrm{wt} \%$. Furthermore, the application to the residue of three successive extraction cycles increased the oil yield up to a maximum of $84.65 \mathrm{wt} \%$ of the total soybean oil recovered in the form of intact oleosomes. ${ }^{60}$ In an attempt to minimize waste, Kapchie et al. ${ }^{71}$ studied the possibility of reusing the resulting aqueous supernatant, which may be rich in sodium chloride and saccharose, for performing aqueous enzymatic or non-enzymatic extraction of oleosomes from fresh soybean flour. Although the yield of oleosome extraction was significantly higher in the first enzymatic extraction (81.41 \pm $2.24 \mathrm{wt} \%)$, the extraction yield (73.09 $\pm 3.39 \mathrm{wt} \%)$ when the supernatant was reused with no additional enzymes was also satisfactory. Soybean oleosomes were recovered by applying an enzyme-assisted aqueous extraction method in a pilot plant exhibiting a very high yield (up to $93.40 \mathrm{wt} \%$ ) which was significantly higher than the yield of the laboratory scale extraction (76.83 wt\%). This was an indication that the application of a large scale oil body isolation process is possible. ${ }^{72}$

\subsection{Recovery of extracted oil bodies}

The recovery of oil bodies from their aqueous extract has been the objective of a number of research studies, aiming at reducing the storage and/or transport volume and extending the range of potential applications of oil bodies in the preparation of novel, end food or other products. A very rich-in-oil droplets cream, practically free from extraneous plant protein impurities, may be obtained from the initial extract by gravitational creaming $^{12}$ and then washing the cream with a saccharose water solution. ${ }^{1,73}$ Chen et al. showed that the yield of oil recovery can be improved by increasing the centrifugation speed up to 30000 rpm. ${ }^{65}$ Another method that has been described by Nikiforidis and Kiosseoglou ${ }^{73}$ is isoelectric aggregation that involves the centrifugation of oil bodies extract after adjusting the $\mathrm{pH}$ of the extract to a value close to 5.0 in order to bring about the 
aggregation of the oil droplets and aid their rise to the top. As reported by the authors, the overall yield of the combined extraction and recovery steps was close to $75.5 \mathrm{wt} \%$, indicating that a significant loss of oil during the application of the recovery step took place, probably because of the inability of all the oil droplets in the extract to aggregate and cream to the top. In addition, the recovered cream was richer in extraneous proteins than the cream obtained by the previous method, probably due to co-aggregation of the oil droplets with a fraction of the exogenous proteins of the extract.

The product of oil droplet recovery from their initial extract by employing techniques based on the principle of gravitational aggregation is always a cream with a degree of concentration in terms of oil content depending on the method applied. In the case of the cream obtained by centrifugation in the presence of saccharose the oil content of the final cream may reach values as high as $90 \mathrm{wt} \%$ on the total weight basis, depending on the centrifugation speed while when isoelectric aggregation of oil bodies is applied the yield value cannot exceed the limit of $40 \mathrm{wt} \%$ (unpublished data). In a completely different approach, concentration of the initially dilute oil body extract by ultrafiltration may be applied, leading not to a rich in-oil cream as the application of the previously described methods, but instead, to a natural emulsion derived from oil bodies with a composition close to that of the commercially available soymilk. ${ }^{74,75}$ Nikiforidis et al. $^{38}$ applied this method to dilute (0.1 wt\% in oil) extract of maize germ oil droplets and exogenous proteins and obtained much more concentrated emulsion with an oil content close to $5.0 \mathrm{wt} \%$ which practically represented almost $100 \%$ of the initially extracted oil mass.

\section{Physical and chemical properties oil body-based natural emulsions}

Initial oil body extracts from various plant sources contain both the endogenous proteins adsorbed at the oil body surface as well as co-extracted with the oil body seed storage proteins, mainly dispersed in the water. ${ }^{76,77}$ However, the final materials, obtained by applying the recovery methods described above may differ considerably both in their oil as well as in protein content and composition. These differences are expected to affect their physical and chemical properties and also determine their usefulness in the development of end food products. Irrespective, however, of their composition, the three oil body preparations (the two types of cream and the emulsion) are all in effect dispersions of intact or disrupted oil bodies in a protein solution. These materials may become physically and/or chemically destabilized during further treatment and/or storage. Aggregation of oil droplets into large aggregates or, what is even more important, coalescence into droplets of a larger size, leading to oil separation at the top of the container, are undesirable changes that have to be halted or at least slowed down when considering the physical stability of food emulsions. The oil droplets may also suffer chemical and, possibly, enzymatic changes during storage and/or processing with the lipid autoxidation reactions being the best well known.
As the presence of exogenous seed proteins may play a key role in determining the stability of oil bodies, a number of researchers have included in their publications analytical data regarding the presence of exogenous proteins in the rich-in-oil preparations recovered from different raw materials. SDS-PAGE is a useful analytical tool to discriminate between the exogenous proteins and those of the oil body surface. As it is clearly presented in Fig. 4 maize germ oil droplets recovered from the initial extract by the method of isoelectric aggregation may contain proteins of a wide variety of sizes, ranging from 15 up to $60 \mathrm{kDa} .{ }^{73}$ On the other hand, as it may be seen in lane 4 , where the electrophoregram of oil droplets recovered from their extract by gravitational creaming in presence of sucrose is illustrated, the surface of the oil bodies consisted almost entirely of oleosins of a molecular size between 15 and $16 \mathrm{kDa}$. Caleosins $(\sim 25 \mathrm{kDa})$ and steroleosins $(\sim 50 \mathrm{kDa})$ are also present but at a much lower concentration.

\subsection{Physical stability}

Since the oil droplets recovered from their initial extract by the method of extensive washing contain practically no exogenous plant proteins at all, the only factors that may affect their physical stability in model oil-in-water emulsions is their size and surface protein content. In general, there is a wide range of sizes found in oil bodies originating from various oleaginous raw materials, related to some extent to the seed oil and oleosin concentration. ${ }^{78,79}$ For example, purified oil droplets recovered from maize germ, ${ }^{\mathbf{8 0}}$ sunflower seeds ${ }^{\mathbf{4 0}}$ and sesame seeds (unpublished data), were reported to have $d_{3.2}$ values around 0.4, 1.0 and 0.7 respectively. In general, emulsions of oil droplets with a larger size are less stable during storage than those with a smaller size..$^{\mathbf{8 1 - 8 3}}$ In addition, natural oil-in-water emulsions obtained from sources like soybean ${ }^{\mathbf{1 2}}$ or maize germ, ${ }^{73}$ with relatively small sizes are more stable than the ones originating from sesame seed with a larger size. ${ }^{\mathbf{8 4 - 8 7}}$

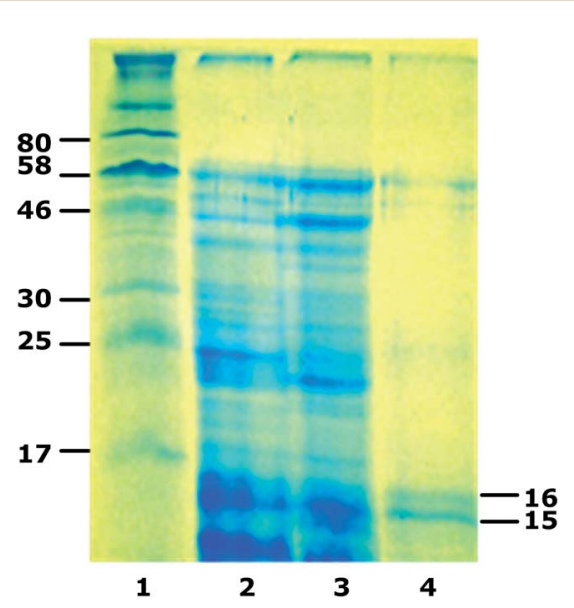

Fig. 4 SDS-PAGE profiles of molecular marker (lane 1), maize germ (lane 2), cream recovered with isoelectric aggregation (lane 3), and washed oil body-based cream (lane 4) proteins. Reprinted with permission from Nikiforidis et al. ${ }^{73}$ 
Highly purified oil droplets in oil body-based emulsions, irrespective of their origin, are prone to aggregate relatively easily, probably because of the relatively weak electrostatic repulsion forces operating between the droplets. ${ }^{88}$ Aggregation of droplets and coalescence that follows, and may then limit their potential for application in foods. Long term stability can be enhanced by applying emulsification, ${ }^{\mathbf{8 7}, 89,90}$ which is not a cost-efficient approach for industrial purposes, or by addition of surfactants and/or biopolymers. ${ }^{73,80,88,91,92}$ Improvement of physical stability of oil droplets may also result from the application of heat treatment $\left(90{ }^{\circ} \mathrm{C}, 30 \mathrm{~min}\right)$ to the initial oil body extract. ${ }^{93}$ The improvement of the long-term stability was attributed to the deactivation of endogenous enzymes such as lipase and lipoxygenase.

Recovery of oil from the initial aqueous extract by isoelectric aggregation is expected to lead to the recovery of oil droplets in the form of a cream enriched in seed storage proteins. ${ }^{3,73}$ Exogenous proteins remain at the oil body surface even after redispersion of the cream in aqueous solutions and the formation of oil-in-water emulsions (Fig. 4). ${ }^{3}$ These proteins may provide additional stability to the oil droplets which is attributed to the secondary layer that forms at their surface, enhancing thus the strength of the repulsive steric forces operating between neighbouring droplets. The emulsions also exhibit high stability against coalescence, even after storage for long time periods. ${ }^{73}$

A natural emulsion obtained from oil body extraction with an oil content of $5 \mathrm{wt} \%$ may be obtained following recovery of the initially extracted oil by applying ultrafiltration to the initial maize germ oil body extract. ${ }^{38}$ The mean surface-volume diameter of this emulsion was $155 \mathrm{~nm}$. This natural nanoemulsion, apart from the interfacial proteins, also contained all the exogenous storage proteins of the extract (Fig. 5) and exhibited a remarkable physical stability even after heating at $90{ }^{\circ} \mathrm{C}$.

\subsection{Physical stability in presence of surfactants or biopolymers}

Since the physical stability of some of the oil bodies-based emulsions may be relatively low, the idea of incorporating

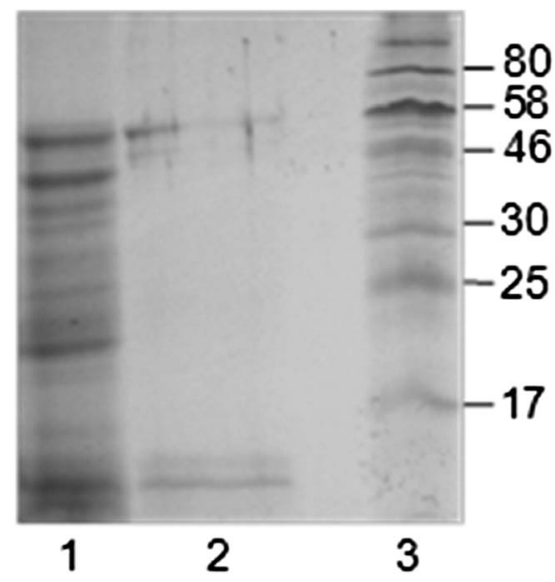

Fig. 5 SDS-PAGE of proteins of oil-body nanoemulsion (lane 1), of the washed oil body-based cream (lane 2) and of the molecular marker (lane 3). Reprinted from Nikiforidis et al. ${ }^{38}$ surfactants or biopolymer molecules into their water phase was put forward by a number of investigators. The stability of a natural emulsion based on maize germ oil bodies is enhanced following addition of Tween 80, especially at a concentration level of $0.75 \% .^{91}$ The improvement in emulsion stability was attributed by the authors to the alteration of the composition of the oil body surface since the addition of the surfactant resulted in the development at the surface of an adsorbed film of a mixed nature, made up of surfactant- and phospholipid-rich domains, with the non-displaced surfactant protein molecules, mainly oleosins, remaining embedded in the latter (Fig. 6). ${ }^{91}$

Surface active biopolymers such as proteins may also be considered as possible oil droplet stabilizers. However, in straight contrast to the surfactants which may competitively adsorb to the oil droplet surface, due to their small size, the problem with the proteins is that even the ones with a very flexible molecular structure may find it hard to displace the natural emulsifiers of the oil bodies and adsorb in their place. For example it was recently reported that in spite of their high surface activity, yolk lipoproteins are not able to penetrate and adsorb to the highly cohesive and hydrophobic mixed phospholipids-protein surface membrane of the maize germ oil bodies. ${ }^{80}$ However, the presence of egg yolk constituents in the emulsion continuous phase led to a marked enhancement of its stability against coalescence. As was suggested by the authors, the presence in the emulsion water phase of non-adsorbed yolk protein particles resulted in the intensification of interdroplet interaction, due to depletion events. Therefore, as it is illustrated in Fig. 7 they might have had an indirect but still strong influence on emulsion structure and physical stability.

In contrast to egg yolk lipoproteins, caseinate molecules can adsorb to the surface of oil droplets of natural emulsions based on isolated maize germ oil bodies and offer extra protection against coalescence. The adsorption takes place when the oil droplets are equilibrated against a caseinate solution under agitation. ${ }^{94}$ According to the SDS-PAGE analysis, the $\beta$ - and $\kappa$ caseins, were the caseins that mainly adsorbed to the surface. ${ }^{95}$

Other additives that could be useful in offering additional protection to natural emulsions are charged polysaccharides such as pectin or xanthan gum. According to recent findings, ${ }^{\mathbf{8 8 , 9 2}}$ pectin is a polysaccharide that enhances the creaming stability

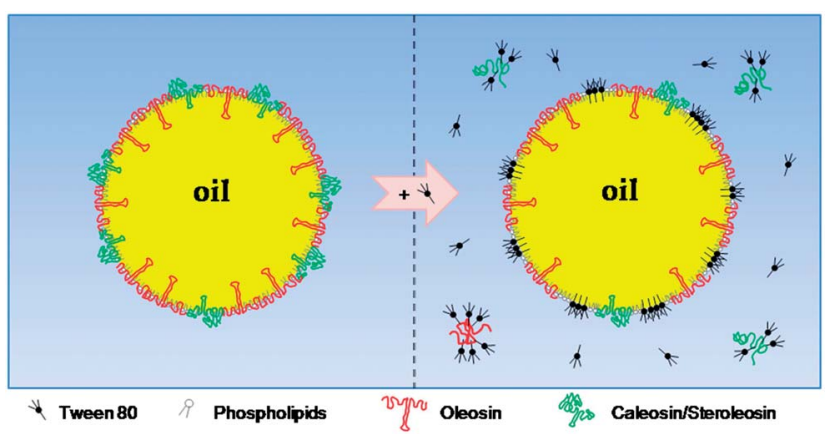

Fig. 6 Schematic representation of the potential changes in oil body surface structure resulting from Tween addition. Reprinted from Nikiforidis et al. ${ }^{91}$ 


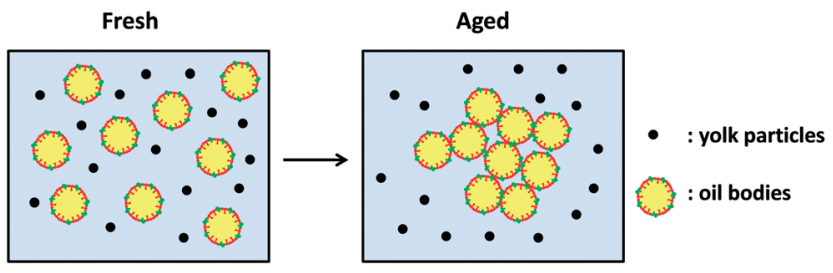

Fig. 7 Schematic representation of structure development with ageing of interactions of purified natural emulsified oil droplets and egg yolk. Reprinted from Nikiforidis et al. ${ }^{80}$

of purified oil droplets extracted from soybeans, something that was attributed to the formation of a secondary layer of polysaccharide molecules at the oil droplet surface through electrostatic interaction with the protein constituents of the primary surface layer.

Enhancement of the physical stability of oil bodies can also be achieved with the use of xanthan gum. ${ }^{73}$ Xanthan gum addition at a concentration of $0.1 \mathrm{wt} \%$ was found to bring about a significant enhancement in the creaming stability of purified oil droplets obtained from maize germ by aqueous extraction. The improvement against creaming was again attributed to the appearance of electrostatic interactions between xanthan gum and the proteins of the oil droplet surface that led to the diminution of depletion flocculation effects and to improvement of the steric repulsive forces.

\subsection{Chemical stability}

In addition to their physical stability, oil bodies in the rich-in oil plant raw materials may also exhibit remarkable stability against lipid oxidation. This is apparently connected with the presence both inside the oil bodies as well as in their perimeter of bioactive phytochemicals with antioxidant activity. ${ }^{96}$ There are also recent research findings suggesting that a number of oil body-based natural emulsions obtained with the use of aqueous media may also exhibit significant oxidative stability., ${ }^{4,59,93,97-100}$ Even artificially-prepared oil bodies turned out to be more resistant to lipid oxidation when compared with technicallyprepared emulsions formulated with surfactants or proteins. ${ }^{101}$ This is of great importance for the development of chemicallystable novel food products based on oil bodies.

The stability of oil against oxidation in natural oil body emulsions derived from oil body aqueous extraction seems to be the result of the presence in the emulsion continuous phase of both the residual seed proteins, along with those present in the mixed phospholipids-proteins surface membrane. It has also been proposed that the presence of antioxidants, like E-vitamers, associated with oat oil body surface may contribute to their oxidative stability. ${ }^{4}$ Moreover, since the initial oil body size is inextricably tied to the size of the surface area, it may also play an important role in the oxidative stability of oil, but it is a factor that is hard to fully control. ${ }^{97}$ On the other hand, extensive heat treatment of the oil droplet suspensions immediately after their extraction of oil bodies from soybean was found to significantly improve their chemical stability. This is attributed to the deactivation of endogenous enzymes such as lipase and lipoxygenase. ${ }^{93}$

Since the method applied for the recovery of oil droplets from the initial oil body extract may have an effect on droplet size and also on the exogenous proteins content of their surface, it is reasonable to assume that the method of recovery is highly crucial in determining the chemical stability of the recovered oil bodies. ${ }^{5}$ As an example, natural emulsions based on oil bodies recovered from a maize germ extract by applying the technique of gravitational creaming in presence of sucrose, exhibited a relatively low stability to lipid oxidation. On the other hand, recovery of oil droplets by isoelectric aggregation produced emulsions exhibiting a much higher stability against oil oxidation..$^{98}$ Moreover, a natural emulsion based on maize germ oil bodies obtained from the initial extract by applying ultrafiltration, exhibited an even higher chemical stability. It seems that the presence of exogenous seed protein molecules in the emulsion continuous phase was the reason for the remarkable oxidative stability of the natural emulsion.

\section{Main potential food applications}

The final preparations obtained by aqueous extraction of oil from oil-rich materials followed by recovery of the extracted oil droplets, may have the form of either a liquid $\mathrm{o} / \mathrm{w}$ natural emulsion or a semi-liquid cream, depending on the recovery method applied. In both the emulsion and the cream the oil is found in the form of relatively small-sized oil bodies dispersed in a plant protein solution The considerable difference in the oil content between the two types of oil body-based preparations is bound to determine their future exploitation as ingredients in the development of a number of food or other products. With regard to foods, a number of potential applications could be the development of imitation milk beverages and other dairy-like products. ${ }^{102,103}$ In addition, many other liquid, semi liquid or solid foods, appearing in the form of an oil-in-water emulsion (e.g. sauces, mayonnaise and salad dressings) or incorporating emulsified oil/fat droplets into a protein and/or polysaccharidebased matrix (e.g. meat, pastry or baked products), constitute another group of interest for potential applications.

The technological/economic advantages of replacing in the various food formulae of technically-emulsified oil/fat with oil bodies are more than obvious and have already been discussed in the introductory section. Incorporation, however, of oil bodies into the structure of a food material is not always as easy as it may sound. In fact, a number of problems may be faced by food technologists involved in product development when attempting to prepare homogeneous mixtures of naturallyemulsified oil droplets with the rest of product ingredients. These problems are mainly connected with possible incompatibility effects between the oil body surface constituents and biopolymeric food ingredients, namely proteins and/or polysaccharides. As a result of such effects, the food structure may differ from that of a traditionally-prepared food product and the novel food may exhibit inferior sensory characteristics and poor quality. Furthermore, following long time product storage, incompatibility phenomena may lead to oil separation. 
The stability against coalescence of natural emulsion obtained from oil body aqueous extraction is another crucial parameter that may determine their applicability in the preparation of novel food or other products. The coalescence of oil droplets into larger ones, eventually leading to the appearance of undesirable oiling-off at the product surface, depends on their initial droplet size and the composition of their surface membrane. In addition, the intensity of thermal treatment, e.g. heat treatment of the oil body-based emulsion or "cream" for pasteurization/sterilization purposes, may also have an effect on the rate of droplet coalescence and the final droplet size. Considering that the heat-treated oil body-based emulsion or "cream" may again suffer further thermal treatment, following incorporation into the initial product mix, the heat stability of the oil droplets against coalescence could be an important parameter that may determine their suitability for certain applications. The same should hold for applications where the end product is stored under freezing conditions, leading to disruption of the stabilizing surface layer and, therefore, droplet coalescence and oil leakage from the food structure. The stability against coalescence of the naturally emulsified oil droplets incorporated into the food structure, may also depend on the extent of phenomena of depletion flocculation and creaming and also on the presence of low molecular weight emulsifiers that antagonize the proteins of the oil droplet surface leading to their desorption and hence to the weakening of the strength of the surface membrane. ${ }^{91}$

A final important parameter that has to be taken into account when contemplating the application of oil bodies in the preparation of foods and possibly other products is the presence of off-flavors, connected with volatile compounds that appear as a result of lipid oxidation. Hexanal, for example, which is a secondary product of lipid oxidation, is one of the compounds responsible for the unpleasant beany flavor of soymilk and has to be masked by incorporating flavouring additives, such as vanillin. Apart from the off-flavours connected with lipid oxidation, unpleasant volatiles may also originate from the oil of the natural emulsion when their initial mix with other product ingredients is subjected to fermentation for the preparation of dairy-like products. As both the oil and the protein constituents of oil droplets are completely different from those of the animal milk, their fermentation with microorganisms to prepare products such as yogurt or probiotic beverages may lead to the appearance of unusual volatiles, possibly unacceptable by the consumer.

\subsection{Dairy-like foods and beverages}

This is one of the main areas of potential applications of natural emulsions and creams based on oil bodies. Products belonging to this category are imitation milk and other beverages, yogurt, cheese, cream, ice cream etc. The process of preparation of a number of products such as yogurt or kefir, always involves fermentation of the mix with the use of microorganisms. The final fermented products are expected to contain viable probiotic microorganisms and may, therefore, be characterized as probiotic foods and beverages, providing that the viable probiotic cell count exceeds a level.

The most widely known imitation dairy product is soymilk prepared by mixing a thermally-treated aqueous extract of soybeans with sugar (sweet soymilk) and flavouring additives. Jacques et al. ${ }^{\mathbf{1 0 4}}$ described the process of preparing a vegetable milk-like beverage based on water extract of oil bodies from almond. A similar product, also based on naturally emulsified oil from pistachio nuts paste, was recently developed by Shakerardekani et $a .^{63}$ In this study a series of beverages were prepared by mixing the initial oil body pistachio nut extract with additives such as vanillin, sugar and salt at various concentrations. As reported by the authors the most preferred beverage of the series as assessed by a group of untrained taste panelists was the one containing $5.0 \mathrm{wt} \%$ sugar, $0.02 \mathrm{wt} \%$ vanillin and 0.0 wt\% salt.

Development of probiotic non-dairy yogurt and beverages by fermenting water extracts of oil bodies from soybean or other sources, with the use of various probiotic Lactobacillus strains has been the subject of a number of papers. ${ }^{105-107}$ In a relatively recent publication, Mishra and Mishra ${ }^{\mathbf{1 0 8}}$ reported that yogurtlike probiotic products with improved textural and flavour characteristics can be produced by fermentation of soymilk, containing the prebiotic fructo-oligosaccharide, with binary combinations of a number of Lactobacillus strains. The best results with regard to acidification profile, product flavour and textural/rheological characteristics were obtained by the combination of L.acidophilus-L.plantarum. In addition to soybean, the aqueous extract of the walnut kernel has also been subjected to fermentation with lactic acid bacteria in an attempt to prepare beverages with probiotic properties. ${ }^{109,110}$ Jing $^{111}$ reported that the viable counts of the probiotic lactococci and lactobacilli in the fermented wall nut extract beverage were around $7.9 \times 10^{7} \mathrm{CFU} \mathrm{ml}^{-1}$. In a more recent paper, ${ }^{105}$ walnut milk containing 6-12 wt $\%$ (w/v) sucrose was fermented with the use of kefir grains and a probiotic beverage was obtained. Based on sensory analysis data it was concluded that the most acceptable product was obtained at a sucrose concentration of $8 \mathrm{wt} \%$ and a fermentation temperature and time of $30^{\circ} \mathrm{C}$ and $12 \mathrm{~h}$, respectively. The viable cell counts surviving in the fermented beverage for lactococci; lactobacilli and yeast were $8.2 \times$ $10^{7}, 1.1 \times 10^{8}$ and $1.0 \times 10^{6} \mathrm{CFU} \mathrm{ml}^{-1}$, respectively, suggesting thus that the kefir grains can be used as a starter culture in the preparation of a walnut milk-based probiotic beverage.

\subsection{Salad dressings and sauces}

This group of food products includes a number of semisolid, oil-in-water emulsions based usually on egg yolk as their main emulsifier and with an oil volume fraction that in salad dressing products such as mayonnaise may reach a value as high as 0.8 or even higher. On the other hand, the oil content of products such as light salad dressings is much lower and incorporation of a polysaccharide at a relatively low concentration is needed to stabilize the emulsion and thicken its texture. Irrespective, however, of the oil content, the successful incorporation of oil into the structure of such products is expected to depend on the 
behaviour of the egg yolk constituents towards the naturally emulsified droplets of the extract since the oil droplets of a traditionally-prepared yolk stabilized-dressing emulsion are covered with a membrane with a composition completely different from that of the oil body surface layer.

Considering that the main proteins of the oil body surface, the oleosins, are highly hydrophobic proteins, it is of interest to know how the relatively hydrophobic apo-lipoproteins of the yolk will behave when coming into contact with the natural oil droplet surface layer. According to the conclusions of a recently published study on model salad dressing emulsions, based on maize germ oil body extracts, with an oil volume fraction of 0.45 or $0.2,{ }^{112}$ the hydrophobic and highly flexible yolk apo-lipovitellin molecules were not able to competitively displace the oleosins of the oil bodies from the surface film and adsorb in their place. Since, however, both the mechanical properties of the emulsions and their physical stability were positively affected by the presence of egg yolk constituents, it was hypothesized by the authors that such effects were connected with the appearance of depletion flocculation phenomena, resulting from the presence of the yolk particles in the emulsion continuous phase. As a result of the droplet flocculation, the weak gel structure of salad dressing emulsion was further reinforced. At the same time, oil droplet reorientation lead to a more compact structure and oil droplet coalescence was halted. This study showed that a stable dressing-type emulsion with improved rheological properties and physical stability can be prepared even though the main emulsifier of the mixture, the egg yolk lipoproteins, are not able to penetrate the natural surface layer of the extracted oil bodies and become adsorbed at the expense of the oleosins. However, it should be born in mind that the droplets of naturally emulsified maize germ oil were of a very small size and may, therefore, have exhibited high coalescence stability when they flocculated with other neighboring oil droplets. Large-sized and hence less stable oil body-based natural emulsions of an origin other than the droplets of naturally emulsified maize germ may not be able to resist the mechanical stresses arising from depletion flocculation, leading thus to the emulsion destabilization.

\subsection{Edible films and coatings}

Incorporation of emulsified oil droplets into the structure of edible films, based on proteins or polysaccharides, is a relatively new area of research aiming at developing food packaging films and coatings with improved water barrier properties and possibly acting as carriers of bioactive hydrophobic compounds, such as antioxidants. The successful incorporation of oil droplets into the film matrix depends on the close interaction of the film-forming biopolymer with the oil droplet surface. Therefore, substitution of oil bodies for technicallyemulsified oil droplets may result in films exhibiting inferior mechanical properties and appearance due to incompatibility between the film-forming polymer molecules and those of the oil body' surface.

An extensive study, where oil body aqueous extracts originating from soybean were applied for developing filled with oil bodies edible films, was conducted by Wang ${ }^{\mathbf{1 1 3}}$ without much success since the mechanical properties of the prepared films were very poor. However, when the oil droplets of a soybean aqueous extract were incorporated into the structure of polysaccharide-based films, the outcome was more satisfactory.

Matsakidou, et al., ${ }^{94}$ also succeeded in preparing sodium caseinate-based composite films, filled with naturally emulsified maize germ oil. The films exhibited a milky appearance which could be useful in certain applications, improved resistance to water vapour permeability and higher surface hydrophobicity when compared with the control films not containing oil bodies. Although some irregularities, attributed to the presence of flocculated oil droplets, were spotted at the film surface the physical properties of the composite films was very satisfactory. The interaction between the protein matrix and the oil droplet surface layer, where a number of the flexible sodium caseinate molecules were adsorbed, was according to the authors because of the homogeneity of the composite films.

\section{Conclusion and future perspectives}

Oil body-based preparations in the form of a natural emulsion or a concentrated "cream" may be obtained from rich-in-oil plant materials by subjecting the comminuted raw material to aqueous extraction and then recovering the extracted naturally emulsified oil by applying a number of different recovery methods. The finally recovered oil body-based preparation may exhibit totally different compositions in terms of oil and proteins as well as in their physical and/or chemical stability. As a result, incorporation of such preparations into the structure of novel food products has to take into account both their stability properties, as well as possible phase separation phenomena appearing between the surface of the extracted oil droplets and the rest of product ingredients, namely the proteins and/or polysaccharides. Finding ways to overcome such obstacles is a prerequisite for the successful development of novel food products based on natural emulsions with accepted by the consumer structural and nutritional properties. To achieve this goal a thorough investigation of both the chemical as well the structural characteristics of oil body-based natural emulsions recovered from different oil-rich sources is needed.

\section{Acknowledgements}

Authors would like to thank the action ARISTEIA/820-OILBODIESEXPLOIT, which was co-financed by Greece and the European Social Fund, in the context of the Operational Program "Education and Lifelong Learning", for financial support.

\section{References}

1 J. T. Tzen and A. H. Huang, J. Cell Biol., 1992, 117, 327-335.

2 K. A. Campbell and C. E. Glatz, J. Agric. Food Chem., 2009, 57, 10904-10912. 
3 M. Moloney, J. Boothe and G. v. Rooijen, Eur. Pat. 6924363, 2002.

4 C. V. Nikiforidis and V. Kiosseoglou, J. Agric. Food Chem., 2009, 57, 5591-5596.

5 D. A. White, I. D. Fisk and D. A. Gray, J. Cereal Sci., 2006, 43, 244-249.

6 I. D. Fisk, D. A. White, M. Lad and D. A. Gray, Eur. J. Lipid Sci. Technol., 2008, 110, 962-968.

7 G. G. Adams, S. Imran, S. Wang, A. Mohammad, M. Samil Kok, D. A. Gray, G. A. Channell and S. E. Harding, Food Chem., 2012, 134, 1919-1925.

8 J. Tzen, Y. Cao, P. Laurent, C. Ratnayake and A. Huang, Plant Physiol., 1993, 101, 267-276.

9 F. Beisson, N. Ferté, S. Bruley, R. Voultoury, R. Verger and V. Arondel, Biochim. Biophys. Acta, Mol. Cell Biol. Lipids, 2001, 1531, 47-58.

10 H. C. Lu, P.-L. Jiang, L. R. C. Hsu, C.-L. Chyan and J. T. C. Tzen, Biosci., Biotechnol., Biochem., 2010, 74, 18411847.

11 P. Jolivet, C. Deruyffelaere, C. 1. Boulard, A. Quinsac, R. 1. Savoire, N. Nesi and T. Chardot, Ind. Crops Prod., 2013, 44, 549-557.

12 D. Iwanaga, D. A. Gray, I. D. Fisk, E. A. Decker, J. Weiss and D. J. McClements, J. Agric. Food Chem., 2007, 55, 8711-8716.

13 NIOSH, International Chemical Safety Cards - n-hexane, April 13, 2000.

14 Associated-Press, in The New York Times, December 9, 1984. 15 http://www.factsonline.nl, January 1, 2014.

$16 \mathrm{EC}$, in $S E G / S U M / 52,1995$.

17 I. W. P. Hubener, Hepaticologia Cermania, Mannheim, 1834.

18 C. M. Gottshe, Nov. Actorum Acad. Caes. Leop.-Carol. Nat. Cur., 1862, 20, 267-289.

19 G. von Holle, Ueber die Zellenbläschen der Lebermoose, Heidelberg, 1857.

20 W. Pfeffer, Flora, 1874, 57, 17-27.

21 K. Pihakask, Protoplasma, 1968, 66, 79-95.

22 K. Muller, Bet. dtsch, bot. Ges., 1939, 57, 326-370.

23 W. Kuster, von, Basel, 1894.

24 C. E. Lohmann, Beih. bot. Centralbl., 1903, 15, 215-256.

25 F. Meyer, Naturwissenschaften, 1920, 8, 888-889.

26 A. J. M. Garjeanne, Flora, 1903, 92, 457-482.

27 J. A. Rest and J. G. Vaughan, Planta, 1972, 105, 245-251.

28 J. L. Harwood, A. Sodja, P. K. Stumpf and A. R. Spurr, Lipids, 1971, 6, 851-854.

29 M. I. Gurr, J. Blades, R. S. Appleby, C. G. Smith, M. P. Robinson and B. W. Nichols, Eur. J. Biochem., 1974, 43, 281-290.

30 C. R. Slack, W. S. Bertaud, B. D. Shaw, R. Holland, J. Browse and H. Wright, Biochem. J., 1980, 190, 551-561.

31 L. Yang, Y. F. Ding, Y. Chen, S. Y. Zhang, C. X. Huo, Y. Wang, J. H. Yu, P. Zhang, H. M. Na, H. N. Zhang, Y. B. Ma and P. S. Liu, J. Lipid Res., 2012, 53, 1245-1253.

32 D. Binns, T. Januszewski, Y. Chen, J. Hill, V. S. Markin, Y. M. Zhao, C. Gilpin, K. D. Chapman, R. G. W. Anderson and J. M. Goodman, J. Cell Biol., 2006, 173, 719-731.

33 D. J. Murphy and J. Vance, Trends Biochem. Sci., 1999, 24, 109-115.
34 R. F. E. Raven and S. E. Eichorn, Biology of Plants, W.H. Freeman and Company Publishers, New York, 7th edn, 2005.

35 D. J. Murphy, Protoplasma, 2012, 249, 541-585.

36 T. C. Walther and R. V. Farese, Annu. Rev. Biochem., 2012, 81, 687-714.

37 V. K. Khor, W. J. Shen and F. B. Kraemer, Curr. Opin. Clin. Nutr. Metab. Care, 2013, 16, 632-637.

38 C. V. Nikiforidis, O. A. Karkani and V. Kiosseoglou, Food Hydrocolloids, 2011, 25, 1122-1127.

39 G. I. Frandsen, J. Mundy and J. T. C. Tzen, Physiol. Plant., 2001, 112, 301-307.

40 C. V. Nikiforidis, V. Kiosseoglou and E. Scholten, Food Res. Int., 2013, 52, 136-141.

41 C. Sarmiento, J. H. E. Ross and D. J. Murphy, Plant J., 1997, 11, 783-796.

42 J. T. L. Ting, K. Y. Lee, C. Ratnayake, K. A. Platt, R. A. Balsamo and A. H. C. Huang, Planta, 1996, 199, 158165.

43 C. Montealegre, C. Esteve, M. C. Garcia, C. Garcia-Ruiz and M. L. Marina, Crit. Rev. Food Sci. Nutr., 2014, 54, 611-624.

44 J. H. E. Ross, J. Sanchez, F. Millan and D. J. Murphy, Plant Sci., 1993, 93, 203-210.

45 J. C. F. Chen and J. T. C. Tzen, Plant Cell Physiol., 2001, 42, 1245-1252.

46 J. T. C. Tzen and A. H. C. Huang, J. Cell Biol., 1992, 117, 327335.

47 A. H. C. Huang, Plant Physiol., 1996, 110, 1055-1061.

48 O. Leprince, A. C. van Aelst, H. W. Pritchard and D. J. Murphy, Planta, 1998, 204, 109-119.

49 P. Jolivet, F. Acevedo, C. Boulard, S. d'Andrea, J. D. Faure, A. Kohli, N. Nesi, B. Valot and T. Chardot, Proteomics, 2013, 13, 1836-1849.

50 L. J. Lin, S. S. K. Tai, C. C. Peng and J. T. C. Tzen, Plant Physiol., 2002, 128, 1200-1211.

51 L. G. Alexander, R. B. Sessions, A. R. Clarke, A. S. Tatham, P. R. Shewry and J. A. Napier, Planta, 2002, 214, 546-551.

52 J. A. Napier, F. Beaudoin, A. S. Tatham, L. G. Alexander and P. R. Shewry, in Incorporation Advances in Plant Pathology, Advances in Botanical Research, ed. J. A. Callow, 2001, vol. 35, pp. 111-138.

53 H. Naested, G. I. Frandsen, G. Y. Jauh, I. Hernandez-Pinzon, H. B. Nielsen, D. J. Murphy, J. C. Rogers and J. Mundy, Plant Mol. Biol., 2000, 44, 463-476.

54 K. C. Rhee, C. M. Cater and K. F. Mattil, J. Food Sci., 1972, 37, 90-93.

55 A. Rosenthal, D. L. Pyle and K. Niranjan, Enzyme Microb. Technol., 1996, 19, 402-420.

56 K. A. Campbell, C. E. Glatz, L. A. Johnson, S. Jung, J. M. N. Moura, V. Kapchie and P. Murphy, J. Am. Oil Chem. Soc., 2011, 88, 449-465.

57 T. Wang, in Vegetable oils in food technology: Composition, properties and uses, ed. D. F. Gunstone, Blackwell Publishing, Oxford, UK, 2002, pp. 24-25.

58 A. Rosenthal, D. L. Pyle and K. Niranjan, Food Bioprod. Process., 1998, 76, 224-230.

59 W. J. Wolf, J. Am. Oil Chem. Soc., 1970, 47, 107-108. 
60 V. N. Kapchie, D. Wei, C. Hauck and P. A. Murphy, J. Agric. Food Chem., 2008, 56, 1766-1771.

61 J. M. L. N. de Moura and L. Johnson, J. Am. Oil Chem. Soc., 2009, 86, 283-289.

62 Y. Chen and T. Ono, J. Agric. Food Chem., 2010, 58, 74027407.

63 A. Shakerardekani, R. Karim and N. Vaseli, J. Food Process. Preserv., 2012, 37, 541-545.

64 V. N. Kapchie, L. T. Towa, C. Hauck and P. A. Murphy, Food Res. Int, 2010, 43, 241-247.

65 B. Chen, D. J. McClements, D. A. Gray and E. A. Decker, Food Chem., 2012, 132, 1514-1520.

66 S. Latif and F. Anwar, Food Chem., 2011, 125, 679-684.

67 Y.-l. Zhang, S. Li, C.-p. Yin, D.-h. Jiang, F.-f. Yan and T. Xu, Food Chem., 2012, 135, 304-308.

68 M. Xie, N. Dunford and C. Goad, J. Am. Oil Chem. Soc., 2011, 88, 2015-2021.

69 J. M. L. N. de Moura, K. Campbell, A. Mahfuz, S. Jung, C. E. Glatz and L. Johnson, J. Am. Oil Chem. Soc., 2008, 85, 985-995.

70 H. Li, C. Song, H. Zhou, N. Wang and D. Cao, J. Am. Oil Chem. Soc., 2011, 88, 809-817.

71 V. Kapchie, L. Towa, C. Hauck and P. Murphy, J. Am. Oil Chem. Soc., 2010, 87, 223-231.

72 L. Towa, V. Kapchie, C. Hauck, H. Wang and P. Murphy, J. Am. Oil Chem. Soc., 2011, 88, 733-741.

73 C. V. Nikiforidis and V. Kiosseoglou, J. Agric. Food Chem., 2010, 58, 527-532.

74 N. Jinapong, M. Suphantharika and P. Jamnong, J. Food Eng., 2008, 84, 194-205.

75 S. K. Giri and S. Mangaraj, Int. J. Food Sci. Technol., 2013, 17.

76 A. J. D. Rodelas, E. S. Regalado, D. B. Bela-ong, R. N. Garcia, A. C. Laurena and E. M. T. Mendoza, Philippine Agr. Sci., 2008, 91, 389-394.

77 M. Millichip, A. S. Tatham, F. Jackson, G. Griffiths, P. R. Shewry and A. K. Stobart, Biochem. J., 1996, 314, 333-337.

78 Z. Y. Hu, X. F. Wang, G. M. Zhan, G. H. Liu, W. Hua and H. Z. Wang, Plant Cell Rep., 2009, 28, 541-549.

79 W. X. Liu, H. L. Liu and L. Q. Qu, Theor. Appl. Genet., 2013, 126, 2289-2297.

80 C. V. Nikiforidis, C. G. Biliaderis and V. Kiosseoglou, Food Chem., 2012, 134, 64-73.

81 G. Azuma, N. Kimura, M. Hosokawa and K. Miyashita, J. Oleo Sci., 2009, 58, 329-338.

82 K. Nakaya, H. Ushio, S. Matsukawa, M. Shimizu and T. Ohshima, Lipids, 2005, 40, 501-507.

83 C. Huck-Iriart, M. S. Alvarez-Cerimedo, R. J. Candal and M. L. Herrera, Curr. Opin. Colloid Interface Sci., 2011, 16, 412-420.

84 C. C. Peng, I. P. Lin, C. K. Lin and J. T. C. Tzen, Biotechnol. Prog., 2003, 19, 1623-1626.

85 A. C. N. Chua, P. L. Jiang, L. S. Shi, W. M. Chou and J. T. C. Tzen, Plant Physiol. Biochem., 2008, 46, 525-532.
86 M. T. Chang, C. R. Chen, T. H. Liu, C. P. Lee and J. T. C. Tzen, J. Sci. Food Agric., 2013, 93, 1516-1519.

87 M. T. Chang, T. R. Tsai, C. Y. Lee, Y. S. Wei, Y. J. Chen, C. R. Chen and J. T. C. Tzen, J. Agric. Food Chem., 2013, 61, 9666-9671.

88 D. Iwanaga, D. Gray, E. A. Decker, J. Weiss and D. J. McClements, J. Agric. Food Chem., 2008, 56, 2240-2245.

89 T. H. Liu, C. L. Chyan, F. Y. Li and J. T. C. Tzen, J. Agric. Food Chem., 2009, 57, 2308-2313.

90 J. M. Tseng, J. R. Huang, H. C. Huang, J. T. C. Tzen, W. M. Chou and C. C. Peng, Biotechnol. Prog., 2011, 27, 153-161.

91 C. V. Nikiforidis and V. Kiosseoglou, Food Hydrocolloids, 2011, 25, 1063-1068.

92 B. C. Chen, D. J. McClements, D. A. Gray and E. A. Decker, J. Agric. Food Chem., 2010, 58, 9259-9265.

93 B. C. Chen, D. J. McClements, D. A. Gray and E. A. Decker, Food Chem., 2012, 132, 1514-1520.

94 A. Matsakidou, C. G. Biliaderis and V. Kiosseoglou, Food Hydrocolloids, 2013, 30, 232-240.

95 C. Holt, J. A. Carver, H. Ecroyd and D. C. Thorn, J. Dairy Sci., 2013, 96, 6127-6146.

96 I. D. Fisk and D. A. Gray, J. Food Sci., 2011, 76, C1349C1354.

97 D. A. Gray, G. Payne, D. J. McClements, E. A. Decker and M. Lad, Eur. J. Lipid Sci. Technol., 2010, 112, 741-749.

98 O. A. Karkani, N. Nenadis, C. V. Nikiforidis and V. Kiosseoglou, Food Chem., 2013, 139, 640-648.

99 N. Nantiyakul, S. Furse, I. Fisk, T. J. Foster, G. Tucker and D. A. Gray, J. Am. Oil Chem. Soc., 2012, 89, 1867-1872.

100 V. N. Kapchie, L. Yao, C. C. Hauck, T. Wang and P. A. Murphy, Food Chem., 2013, 141, 2286-2293.

101 C. Wijesundera, T. Boiteau, X. Q. Xu, Z. P. Shen, P. Watkins and A. Logan, J. Food Sci., 2013, 78, C1340-C1347.

102 S. Gallier, K. C. Gordon and H. Singh, Food Chem., 2012, 132, 1996-2006.

103 H. M. Deckers, G. v. Rooijen, J. Boothe, J. Goll, S. Mahmoud and M. Moloney, US Pat. US6146645A, 2000.

104 B. Jacques, B. Guilaine and B. Martine, Trends Food Sci. Technol., 1997, 8, 62.

105 X.-H. Cui, S.-J. Chen, Y. Wang and J.-R. Han, LWT-Food Sci. Technol., 2013, 50, 349-352.

106 D. I. Serrazanetti, M. Ndagijimana, C. Miserocchi, L. Perillo and M. E. Guerzoni, Food Control, 2013, 34, 336-346.

107 S. Y. Lee, C. V. Morr and A. Seo, J. Food Sci., 1990, 55, 532536.

108 S. Mishra and H. N. Mishra, Food Bioprocess Technol., 2013, 6, 3166-3176.

109 Y. X. Hou, J. H. Xing and L. Liu, J. Henan Univ. Technol., Nat. Sci. Ed., 2008, 29, 72-74.

110 G. Wang, Food Eng., 2010, 1, 17-18.

111 S. Q. Jing, Food Ferment. Ind., 2006, 32, 157-159.

112 C. V. Nikiforidis, C. G. Biliaderis and V. Kiosseoglou, Food Chem., 2012, 134, 64-73.

113 L. Wang, Ph.D. thesis, Purdue University, 2004. 